\title{
2-GROUPS OF ALMOST MAXIMAL CLASS: CORRIGENDUM
}

\author{
RODNEY JAMES
}

(Received 17 August 1982)

Communicated by D. E. Taylor

On page 354 of James (1975) (in the proof of Theorem 5.3(b)) there appear the following two sentences:

"When $n \geqslant 7$, replace $s_{1}$ by $s_{1} s_{n-5}^{\delta}$ and $s_{2}$ by $s_{2} s_{n-4}^{\delta}$. Since $\left(s_{2} s_{n-4}^{\delta}\right)^{2}=s_{2}^{2} s_{n-2}^{\delta}$, we may suppose $\delta=0$."

Unfortunately, the second sentence does not follow from the first. In fact, the first sentence also forces us to replace $s_{4} s_{5}$ by $s_{4} s_{5} s_{n-2}^{8}$ and so $s_{2}^{2}\left(s_{4} s_{5}\right)^{-1}$ remains unaltered. Thus the case $\delta=1$ is omitted in the paper.

If $\delta=1$, then (replacing $s$ by $s s_{1}^{\alpha}$ ) we may suppose $\alpha=0$ and so $s^{4}=1$. It is now easy to establish that we may take $s_{1}^{2}=s_{n-3}, s_{n-2}$ or 1 giving 3 more groups.

Thus, the number of groups of order $2^{n}$ and class $n-2$ is

(i) 29 when $n=7$

(ii) $27+4(n, 2)$ when $n>7$.

The extra three groups were discovered using a computer version of the nilpotent quotient algorithm as described by M. F. Newman (1977).

\section{References}

Rodney James (1975), '2-groups of almost maximal class', J. A ustral. Math. Soc. Ser. A 19, 343-357.

M. F. Newman (1977), 'Determination of groups of prime-power order', Group Theory, Canberra 1975, pp. 73-84 (Proc. Miniconf. Australian National University, 1975. Lecture Notes in Mathematics 573, Springer-Verlag).

School of Mathematics

University of New South Wales

Kensington, N.S.W. 2033

(C) 1983 Australian Mathematical Society $0263-6115 / 83 \$ A 2.00+0.00$ 